\title{
NKCC1 involvement in the epithelial-to-mesenchymal transition is a prognostic biomarker in gliomas
}

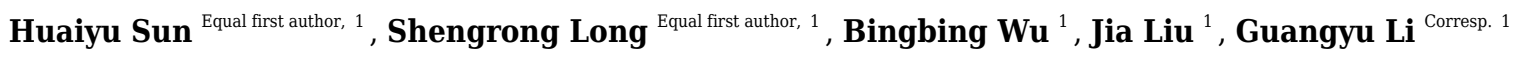 \\ ${ }^{1}$ Department of Neurosurgery, First Hospital of China Medical University, Shenyang, China \\ Corresponding Author: Guangyu Li \\ Email address: liguangyu1972@sina.com
}

Background: Gliomas are the most prevalent type of intracranial tumors. NKCC1 is an important regulator in tumor cell volume. We noticed that abnormally high NKCC1 expression resulted in changes in the shape and adhesion of glioma cells. However, little is known about the role of NKCC1 in the epithelialmesenchymal transition (EMT) of gliomas. This study aims to clarify the biological function of NKCC1 in glioblastoma multiforme (GBM) progression.

Methods: Using data from The Cancer Genome Atlas (TCGA), we performed a Kaplan-Meier analysis on $\mathrm{NKCCl}$ expression levels to estimate the rate of survival of mesenchymal GBM patients. The correlation between NKCC1 and EMT-related proteins was analyzed from the Gene Expression Profiling Interactive Analysis (GEPIA) server. We conducted Gene Set Enrichment Analysis (GSEA) to verify molecular signatures and pathways. We then studied the expression of NKCCI in grade I-IV glioma tissue samples collected from patients using immunohistochemistry (IHC). Finally, we evaluated the effects of NKCC1 migration and invasion on the cellular behaviors of U251 cells using the transwell assay and western blots.

Results: High NKCCl expression was associated with poor prognoses in mesenchymal GBM. Our results suggest a correlation between NKCCI and EMT-protein markers: CDH2 and VIM. GSEA showed that gliomas, TGF-beta signaling and EMT were enriched in the NKCC1 high expression phenotype. Higher expression levels of NKCC1 in gliomas correlate with higher glioma grades. Transwell assay and western blot results demonstrated that the knockdown of NKCC1 led to a reduction in migration and invasion, while also inhibiting MMP-2 and MMP-9 expression in U251.

Conclusion: These results suggest that high expression of NKCC1 regulates EMT in gliomas, providing a new therapeutic strategy for addressing the spread of gliomas by inhibiting the spread of intracranial tumors. 
1 NKCC1 involvement in the epithelial-to-mesenchymal

2 transition is a prognostic biomarker in gliomas

3 Huaiyu Sun ${ }^{1,}$, , Shengrong Long1, *, Bingbing Wu', Jia Liu', Guangyu Li ${ }^{1}$

${ }^{1}$ Department of Neurosurgery, First Affiliated Hospital of China Medical University, Shenyang, China 110001

5 * These authors contributed equally to this work.

7 Correspondence to:

8 Guangyu Li, $\mathrm{PhD}$ MD

9 Department of Neurosurgery

10 First Hospital of China Medical University

$11 \quad 155 \square$ Nanjingbei Street Heping District

12 Shenyang, China 110001

Tel: 13998156450 
21 Abstract

Background: Gliomas are the most prevalent type of intracranial tumors. NKCC1 is an important regulator in tumor cell volume. We noticed that abnormally high NKCC1 expression resulted in changes in the shape and adhesion of glioma cells. However, little is known about the role of $\mathrm{NKCC} 1$ in the epithelial-mesenchymal transition (EMT) of gliomas. This study aims to clarify the biological function of $\mathrm{NKCC} 1$ in glioblastoma multiforme (GBM) progression.

Methods: Using data from The Cancer Genome Atlas (TCGA), we performed a Kaplan-Meier analysis on NKCC1 expression levels to estimate the rate of survival of mesenchymal GBM patients. The correlation between NKCC1 and EMT-related proteins was analyzed from the Gene Expression Profiling Interactive Analysis (GEPIA) server. We conducted Gene Set Enrichment Analysis (GSEA) to verify molecular signatures and pathways. We then studied the expression of NKCC1 in grade I-IV glioma tissue samples collected from patients using immunohistochemistry (IHC). Finally, we evaluated the effects of NKCC1 migration and invasion on the cellular behaviors of U251 cells using the transwell assay and western blots.

Results: High NKCC1 expression was associated with poor prognoses in mesenchymal GBM. Our results suggest a correlation between NKCC1 and EMT-protein markers: CDH2 and VIM. GSEA showed that gliomas, TGF-beta signaling and EMT were enriched in the NKCC1 high expression phenotype. Higher expression levels of NKCC1 in gliomas correlate with higher glioma grades. Transwell assay and western blot results demonstrated that the knockdown of NKCC1 led to a reduction in migration and invasion, while also inhibiting MMP-2 and MMP-9 expression in $\mathrm{U} 251$.

Conclusion: These results suggest that high expression of NKCC1 regulates EMT in gliomas, providing a new therapeutic strategy for addressing the spread of gliomas by inhibiting the spread of intracranial tumors. 


\section{Introduction}

Gliomas are one of the most lethal brain tumors. They affect cognitive function and have a high risk of recurrence, severely affecting quality of life. According to the World Health Organization (WHO), gliomas are classified into grades I-IV. Grades I-II are low-grade gliomas (LGG) and grades III-IV are high-grade gliomas. Among high-grade gliomas, glioblastoma multiforme (GBM) has the highest degree of malignancy and its median survival time is approximately one year [1,2]. Currently, the primary treatment for gliomas is surgical resection followed by radiotherapy, chemotherapy, immunotherapy, photodynamic therapy and electric field therapy [3]. Despite current treatments, GBM has a high rate of recurrence and a 5-year survival rate of $9.8 \%$ [4]. To improve the prognosis of GBM patients, it is important to identify therapeutic targets that effectively prevent intracranial invasion and tumor proliferation.

We can attribute the high recurrence of GBM to the migration of treatment resistant brain-tumorinitiating cells $[5,6]$. During cell migration, GBM cells change their volume to pass through narrow spaces [7]. These volume changes are mediated by ion co-transporters, including the potassium chloride co-transporter, $\mathrm{NKCC} 1$. NKCC1 regulates intracellular volume and $\mathrm{Cl}^{-}$accumulation, allowing $\mathrm{Na}^{+}, \mathrm{K}^{+}$and $\mathrm{Cl}^{-}$to move through the plasma membrane using energy produced by the plasma membrane.

Tumor metastasis is a multi-step process that begins with the tumor cells exiting their primary site to invade healthy cells. Epithelial-mesenchymal transition (EMT) is a crucial process in tumor invasion [8]. EMT is defined by a loss of polarity, tight junctions and adhesion between epithelial cells, resulting in cell infiltration and migration. This process involves changes in the characteristics and morphology of mesenchymal cells [9]. Epithelial cells play an important role in embryonic development, tissue repair, tumor progress and organ fibrosis. During tumorigenesis, there is a higher rate of EMT in migrating cells [10]. 
In our study, we hypothesized that high levels of NKCC1 expression in gliomas were positively correlated with the expression of EMT-related markers: CDH2 and VIM. We concluded that a high expression of NKCC1 was related to gliomas, MAPK signaling pathways, TGF-beta signaling, EMT and other pathways and phenotypes. Our experiments showed that NKCC1 tumor expression increased as the tumor grade increased. NKCC1 knockdown reduced the expression of EMT markers. Simultaneously, glioma invasion was significantly weakened in vitro. These results suggest that $\mathrm{NKCC} 1$ may serve as a therapeutic target inhibiting the migration and invasion of gliomas.

\section{Materials and Methods}

\section{Survival analysis of NKCC1 and its correlation with EMT marker gene expression}

We used Gene Expression Profiling Interactive Analysis (GEPIA) (http://gepia.cancerpku.cn/), an interactive website based on The Cancer Genome Atlas (TCGA), and Genotype-Tissue Expression (GTEx) project data for differential expression analysis, correlation analysis, patient survival analysis, similar gene detection and dimension analysis [11]. Using the GEPIA database, we divided the mesenchymal subtype of GBM into two groups based on the degree of NKCC1 expression and plotted Kaplan-Meier (KM) survival curves. Using GEPIA, a Pearson correlation test was conducted to evaluate the relationship between NKCC1 and EMT proteins in the TCGAGBM datasets.

\section{Gene set enrichment analysis (GSEA)}

GSEA is a computational method used to determine whether a set of previously defined genes show statistically significant and consistent differences between two biological states [12]. We used GSEA to generate an ordered list of genes based on their correlation to NKCC1 expression and to identify survival differences between the high-expression and low-expression of NKCC1. The genes were arranged 100 times per analysis. The expression level of NKCC1 was used as a phenotypic marker. We used the normalized enrichment score (NES) to rank the pathways in each 
102

103

104

105

106

107

108

109

110

111

112

113

114

115

116

117

118

119

120

121

122

123

124

125

126

127

128

phenotype. Analysis was conducted using default settings. A false discovery rate (FDR) of $<0.25$ and NOM p-value of $<0.1$ were considered statistically significant.

\section{Immunohistochemistry}

Glioma tissue samples were collected from the First Hospital of China Medical University. This study was approved by the First Hospital of China Medical University ethics committee (IRB No: 2017-98-2). The patients we chose for this study met the following criteria: a. diagnosed with a primary glioma, b. the patient information is complete and contains clinicopathological and prognostic characteristics. All patients provided written informed consent. We used IHC to detect the expression of NKCC1 in paraffin-embedded glioma tissues. The incubation of primary antibodies (No. ab59791 rabbit-anti-NKCC1 WB 1:1000 IHC 1:50) was conducted overnight at $4^{\circ} \mathrm{C}$. The incubation of secondary antibodies was conducted for two hours at room temperature. We used the VECTASTAIN Elite ABC staining system for immune detection. Diaminobezidin (DAB) was used as the substrate for color visualization. Images were obtained using a Nikon TE2000 Brightfield microscope.

\section{Knockdown of NKCC1}

NKCC1 shRNA (shNKCC1 target sequence, 5'-ACACACTTGTCCTGGGATT-3') and a nontargeting control shRNA sequence were obtained from Takara Biotechnology in Dalian, China. The shRNA sequence was inserted into pRNA-H1.1 to construct pRNA-H1.1-NKCC1 and pRNAH1.1-control plasmids. GBM cell lines U251/U87 were obtained from the cell bank of the Shanghai Institutes for Biological Sciences, Chinese Academy of Sciences. The Short Tandem Repeat (STR) profiles for these two cell lines are provided in the supplementary files. U251 and U87 cells were transfected with pRNA-H1.1-NKCC1 or pRNA-H1.1-control using Lipofectamine 3000 (Invitrogen). The cells were divided into three groups: a. NC group, control U251/U87 cells; b. Vector group, U251/U87 cells transfected with pRNA-H1.1-control plasmids; c. shNKCC1 group, U251/U87 cells transfected with pRNA-H1.1-NKCC1. These cells were collected for 
129

130

131

132

133

134

135

136

137

138

139

140

141

142

143

144

145

146

147

150

151

152

153

154

155

additional experiments 48 hours after transfection. The expression of NKCC1 in U251/U87 cells were analyzed using western blot as described below.

\section{Western blot}

Cellular protein was extracted using a protein extraction kit, according to the manufacturer's instructions (Catalog No. WLA019, Wanleibio, China). Proteins were separated using 8\%-15\% SDS-PAGE gel electrophoresis and transferred to polyvinylidene fluoride (PVDF) membranes. The membranes were blocked with 3\%-5\% bovine serum albumin (BSA) for an hour at $37^{\circ} \mathrm{C}$. The primary antibodies (N-cadherin rabbit [No. ab18203 WB 1:1000; VIM rabbit] [No. ab45939 WB 1:1000; ZEB1 mouse] [No. ab181451 WB 1:1000; MMP-2 rabbit] [No. ab37150 WB 1:1000; MMP-9 rabbit] [No. ab 38898 WB 1:1000; CTNNB1 rabbit] [No. ab16051 WB 1:1000; $\beta$-actin mouse] [No. ab8227 WB 1:500]) were incubated at $4^{\circ} \mathrm{C}$ overnight. The following day, PVDF membranes were washed three times using tris-buffered saline tween-20 (TBST). The PVDF membranes were then incubated with a secondary antibody (goat anti-rabbit/mouse IgG 1:2000) for one hour at room temperature and washed three times, using TBST for five minutes each. Finally, Electrochemiluminescence (ECL) and a gel scanner were used to expose bands of PVDF.

\section{Statistical analysis}

All data were expressed as mean $\pm \mathrm{SD}$ for three independent experiments performed in triplicate; $\mathrm{p}<0.05$ was considered statistically significant. We performed analysis using Statistical Product and Service Solutions (SPSS) 23 and GraphPad Prism 7.0.

\section{Results}

The correlation of NKCC1 with mesenchymal GBM and EMT-related proteins with NKCC1 in GBM

Kaplan-Meier survival analysis showed that the prognosis of GBM in the NKCC1-HIGH group was poor compared to the NKCC1-LOW group $(\log -\operatorname{rank} \mathrm{p}=0.068$; Figure 1$)$. Univariate analysis 
156

157

158

159

160

161

162

163

164

165

166

167

168

169

170

171

172

173

174

175

176

177

178

179

180

181

182

showed that the NKCC1-HIGH expression group was associated with poor overall survival (OS) (hazard ratio [HR]: 1.9). To study the regulatory mechanism of NKCC1 and EMT in GBM, the correlation between the expression of $\mathrm{NKCC} 1$ and EMT markers was analyzed using the GEPIA database. As predicted, NKCC1 expression in the TCGA-GBM dataset positively correlated with EMT-related proteins, $\mathrm{CDH} 2$, the interstitial marker N-cadherin encoding gene, VIM, Zeb1 and CTNNB1 (beta catenin; Figure 2). We performed GSEA on low- and high-NKCC1 expression datasets to determine the differentially activated signaling pathway in GBM. There were significant differences in the enrichment of the MSigDB Collection (Hall Mark and KEGG by GSEA). We selected the most significant enriched signaling pathways using NES. We found that gliomas, MAPK signaling pathways, TGF-beta signaling, EMT and other pathways and phenotypes correlated to a high expression of NKCC1. DNA repair, base excision repair, mismatch repair and nucleotide excision repair correlated to a low expression of NKCC1 (Figure 3).

\section{Immunohistochemistry}

We used IHC to measure NKCC1 expression in glioma tissues. We found that NKCC1 was highly expressed in high-grade (III and IV) gliomas compared to low-grade (I and II) gliomas (Figure 4). Table 1 shows the clinicopathological characteristics of patients with gliomas based on their NKCC1 expression status. These findings show that NKCC1 expression levels are related to different glioma grades $(\mathrm{p}<0.05)$.

\section{NKCC1 is involved in EMT, invasion and migration of U251 and U87 cells}

With a transwell assay we determined the role of $\mathrm{NKCC} 1$ in the invasion of U251 and U87 cells using shRNA-transfected U251/U87 cells and control shRNA U251/U87 cells. We counted the number of cell invasions using Matrigel as the reconstituted basement membrane matrix. We cultured cells under conventional conditions and removed them after 24 hours. We found that migration was significantly lower in shRNA U251/U87 cells than in the control, regardless of the presence of Matrigel (Figure 5). U251/U87 cells were cultured at $37^{\circ} \mathrm{C}$ and $5 \% \mathrm{CO}_{2}$. We 
constructed short hairpin (SH) RNA and found that it knocked down the expression of NKCC1 in U251/U87 cells. Using western blot analysis to silence NKCC1 generated lower expression of EMT markers, MMP2 and MMP9 in U251/U87 cells, compared to the control group. These results suggest that the invasion and migration of U251/U87 cells was inhibited by NKCC1 (Figure 6). CTNNB1 protein expression was also significantly decreased in shNkcc1-U87/U251 cells (Figure 7; * indicates p value $<0.05)$.

\section{Discussion}

Previous studies have reported on the expression and function of NKCC1 in cancer [13-15]. High $\mathrm{NKCC} 1$ expression levels play a role in regulating EMT in gliomas, providing a new therapeutic strategy for addressing the spread of gliomas and inhibiting the spread of intracranial malignancies. The prognostic value of $\mathrm{NKCC} 1$ in GBM has not been explored, leading us to focus on the role of NKCC1 in GBM invasion and migration. Using RNA-seq data from TCGA, our bioinformatics analysis showed that high NKCC1 expression in mesenchymal GBM was associated with a lower survival time and a poor prognosis. To further study the role of NKCC1 in GBM, we used the TCGA-GBM dataset for GSEA, and found that a high expression of NKCC1 was associated with gliomas, MAPK signaling pathways, TGF-beta signaling, and EMT. We also found that in GBM, NKCC1 expression positively correlated with EMT marker expression. IHC showed that NKCC1 was highly expressed in gliomas. We found that higher glioma grades correlated with higher NKCC1 expression. Additionally, western blot analysis revealed that the knockdown of NKCC1 expression levels in U251 decreased EMT-related gene expression. Finally, cell invasion assays revealed that the knockdown of $\mathrm{NKCC} 1$ significantly reduced glioma invasion and migration. The data suggests that NKCC1 may promote EMT in gliomas. CTNNB1 plays an important role in the Wnt/ $\beta$-catenin pathway and contributes to EMT in multiple cancers. EMT is regulated by multiple transcription factors including Snail/2, ZEB1/2, twist1/2 and signaling pathways including TGF $\beta$, ERK1/2, AKT, Notch and WNT/catenin. Therefore, we hypothesize that NKCC1 uses Wnt/ $\beta$ catenin to promote glioma invasion. 
211 NKCC1 is highly expressed in many forms of cancer, including GBM. The high expression of

$212 \mathrm{NKCC} 1$ is often associated with a poor prognosis in GBM patients [16]. In our study, the high

213 expression of NKCC1 was observed in both GBM and U251/U87 cells; high mRNA levels of 214 NKCC1 were associated with a poor clinical outcome in mesenchymal GBM patients. The direct 215 involvement of NKCC1 in biological processes has not yet been studied. We confirmed the 216 relationship between NKCC1 and EMT in gliomas using GSEA. The high expression of NKCC1 217 promoted EMT metastasis by activating the STAT3 signaling pathway in non-small-cell lung cancer [17]. Although GBM is characterized by local invasion, it rarely produces clinically significant extracranial metastasis and only $0.4 \%$ of GBM patients experience metastasis to internal organs. However, there are $20 \%$ of GBM patients with detectable levels of tumor cells circulating in their blood [18]. Additionally, circulating tumor cells from patients diagnosed with metastatic breast cancer showed different epithelial and mesenchymal phenotypes. Circulatig tumor cells (CTCs) exhibited higher levels of interstitial phenotypes than cancer cells within primary tumors [19]. Our results suggest that mesenchymal transition is a key molecular event increasing the malignancy of gliomas [20].

$\mathrm{NKCC} 1$ is found in the tissues of many different animals and is important in multiple physiological functions, including transporting ions to secrete and absorb epithelial cells, maintaining and regulating cell volume, ion concentration and regulating cell growth and development. The mRNA expression of $\mathrm{NKCC} 1$ in the central nervous system is 5 to 40 times higher than in other tissues [21]. Emerging evidence suggests that $\mathrm{NKCC} 1$ plays an important role in changing cell volume during mitosis. NKCC1 blockers can significantly inhibit the proliferation of glioma cells [22]. $\mathrm{NKCC} 1$ is also involved in the process of tumor apoptosis induced by chemotherapeutic drugs and plays an important role in the survival of glioma cells [23]. However, NKCC1's primary function in tumors is to promote call invasion. Existing evidence suggests that NKCC1 changes the adhesion of cells. The regulation of cell volume requires the movement of water, induced by 
237

238

239

240

241

242

243

244

245

246

247

248

249

250

251

252

253

254

255

256

257

258

259

260

261

262

263

osmotic pressure gradients, to achieve regulatory volume reduction (regulatory volume decrease, RVD) and regulatory volume increase (RVI). Chloride ions play an important role in this process, and NKCC1 is one of the most important transporters involved [24]. Some studies have shown that the inhibition of WNK kinase and OSR1 kinase upstream of NKCC1 can also reduce intracellular chloride concentration and inhibit the process of RVI in glioma cells, inhibiting cell invasion [24].

We found that a high expression of NKCC1 promotes EMT in GBM. Many studies support our hypothesis $[25,26]$. We identified a correlation between NKCC1 expression, the TGF- $\beta$ signaling pathway and MAPK signaling pathways using GSEA. However, we did not determine a definitive and specific regulatory relationship or potential mechanism between NKCC1 and these two pathways when we induced EMT in vivo or vitro experimental conditions. This was one limitation of our study.

\section{Conclusion}

NKCC1 promotes migration and invasion of U251/U87 cells. We found that NKCC1 promotes EMT in gliomas. Thus, NKCC1 may act as a potential target for the treatment of malignant gliomas. Other NKCC1 inhibitors that cross the blood-brain barrier may block the NKCC1promoted EMT process in the brain; these may be used in combination with temozolomide to block the invasion and migration of gliomas.

(1)

\section{References}

1. Chen R, Smith-Cohn M, Cohen AL, Colman H. Glioma Subclassifications and Their Clinical Significance. Neurotherapeutics, 2017; 14(2): 284-297.

2. Louis DN, Ohgaki H, Wiestler OD, Cavenee WK, Burger PC, Jouvet A, Scheithauer BW, Kleihues P. The 2007 WHO classification of tumours of the central nervous system. Acta Neuropathol, 2007; 114(2): 97-109. 
264 3. Louis DN, Perry A, Reifenberger G, von Deimling A, Figarella-Branger D, Cavenee WK, 265 Ohgaki H, Wiestler OD, Kleihues P, Ellison DW. The 2016 World Health Organization 266 classification of tumors of the central nervous system: a summary. Acta Neuropathol, 2016 267 ;131(6): 803-820.

268 4. Shen D, Guo CC, Wang J, Qiu ZK, Sai K, Yang QY, Chen YS, Chen FR, Wang J, Panasci $\mathrm{L}$, Chen ZP. Interferon- $\alpha / \beta$ enhances temozolomide activity against MGMT-positive glioma stem-like cells. Oncol Rep, 2015; 34(5): 2715-21.

5. Guerrero Cazares H, Attenello FJ, Noiman L, Quinones-Hinojosa A. Stem cells in gliomas. Handb Clin Neurol, 2012; 104: 63-73.

6. Lathia JD, Mack SC, Mulkearns Hubert EE, Valentim CL, Rich JN. Cancer stem cells in glioblastoma. Genes Dev, 2015; 29(12): 1203-17.

7. Watkins S, Sontheimer H. Hydrodynamic cellular volume changes enable glioma cell invasion. J Neurosci, 2011; 31(47): 17250-9. 8. Lemieux E, Bergeron S, Durand V, Asselin C, Saucier C, Rivard N. Constitutively active MEK1 is sufficient to induce epithelial-to-mesenchymal transition in intestinal epithelial cells and to promote tumor invasion and metastasis. Int J Cancer, 2009; 125: 1575-86. 9. Brandhagen BN, Tieszen CR, Ulmer TM, Tracy MS, Goyeneche AA, Telleria CM. Cytostasis and morphological changes induced by mifepristone in human metastatic cancer cells involve cytoskeletal filamentous actin reorganization and impairment of cell adhesion dynamics. BMC Cancer, 2013; 13: 35.

10. Zhan L, Chen LY, Chen Z, Knockdown of FUT3 disrupts the proliferation, migration, tumorigenesis and TGF- $\beta$ induced EMT in pancreatic cancer cells. Oncol Lett, 2018; 16: 924-30. 11. Tang Z, Li C, Kang B, Gao G, Zhang Z. GEPIA: A web server for cancer and normal gene expression profiling and interactive analyses. Nucleic Acids Res, 2017; 45(W1): W98-W102. 12. Subramanian A, Tamayo P, Mootha VK, Mukherjee S, Ebert BL, Gillette MA, Paulovich A, Pomeroy SL, Golub TR, Lander ES, Mesirov JP. Gene set enrichment analysis: a knowledgebased approach for interpreting genome-wide expression profiles. Proc Natl Acad Sci U S A, 2005; 102(43): 15545-50.

13. Sun PL, Jin Y, Park SY, Kim H, Park E, Jheon S, Kim K, Lee CT, Chung JH. Expression of

$\mathrm{Na}+-\mathrm{K}+-2 \mathrm{Cl}$ - cotransporter isoform $1(\mathrm{NKCC} 1)$ predicts poor prognosis in lung adenocarcinoma and EGFR-mutated adenocarcinoma patients. QJM, 2016; 109: 237-44. 
298

299

300

301

302

303

304

305

306

307

308

309

310

311

312

313

314

315

316

317

318

319

320

321

322

323

324

325

326

327

328

329

330

331

20: 6844-59.

15. Wright PK, May FE, Darby S, Saif R, Lennard TW, Westley BR. Estrogen regulates vesicle trafficking gene expression in EFF-3, EFM-19 and MCF-7 breast cancer cells. Int J Clin Exp Pathol, 2009; 2: 463-75.

16. Pavón MA, Arroyo-Solera I, Téllez-Gabriel M, León X, Virós D, López M, Gallardo A, Céspedes MV, Casanova I, López-Pousa A, Mangues MA, Quer M, Barnadas A, Mangues R. Enhanced cell migration and apoptosis resistance may underlie the association between high SERPINE1 expression and poor outcome in head and neck carcinoma patients. Oncotarget, 2015; 6(30): 29016-33.

17. Lin X, Lin BW, Chen XL, Zhang BL, Xiao XJ, Shi JS, Lin JD, Chen X. PAI1/PIAS3/Stat3/miR-34a forms a positive feedback loop to promote EMT-mediated metastasis through Stat3 signaling in Non-small cell lung cancer. Biochem Biophys Res Commun, 2017; 493(4): 1464-1470.

18. Awan M 1, Liu S, Sahgal A, Das S, Chao ST, Chang EL, Knisely JP, Redmond K, Sohn JW, Machtay M, Sloan AE, Mansur DB, Rogers LR, Lo SS. Extra-CNS metastasis from glioblastoma: A rare clinical entity. Expert Rev of Anticancer Ther, 2015 May; 15(5): 545-52. 19. Yu M 1, Bardia A, Wittner BS, Stott SL, Smas ME, Ting DT, Isakoff SJ, Ciciliano JC, Wells MN, Shah AM, Concannon KF, Donaldson MC, Sequist LV, Brachtel E, Sgroi D, Baselga J, Ramaswamy S, Toner M, Haber DA, Maheswaran S. Circulating breast tumor cells exhibit dynamic

changes in epithelial and mesenchymal composition. Science, 2013; 339 (6119): 580-4.

20. Kahlert UD, Nikkhah G, Maciaczyk J. Epithelial-to-mesenchymal(-like) transition as a relevant molecular event in malignant gliomas. Cancer Lett, 2013; 331(2): 131-8.

21. TR Yerby, CR Vibat, D Sun, JA Payne, ME O'Donnell. Molecular characterization of the $\mathrm{Na}-\mathrm{K}-\mathrm{Cl}$ cotransporter of bovine aortic endothelial cells. American Journal of Physiology, 1997 , 273 (1 Pt 1) :C188.

22 Turner KL, Sontheimer H. Cl- and $\mathrm{K}+$ channels and their role in primary brain tumour biology. Philos Trans R SocLond B Biol Sci. 2014. 369(1638): 20130095.

23 Algharabil J, Kintner DB, Wang Q, Begum G, Clark PA, Yang SS, Lin SH, Kahle KT, Kuo JS, Sun D. Inhibition of $\mathrm{Na}(+)-\mathrm{K}(+)-2 \mathrm{Cl}(-)$ cotransporter isoform 1 accelerates temozolomidemediated apoptosis in glioblastoma cancer cells. Cell Physiol Biochem, 2012;30(1):33-48. 24 Zhu W, Begum G, Pointer K, Clark PA, Yang SS, Lin SH, Kahle KT, Kuo JS, Sun D. WNK1-OSR1 kinase-mediated phospho-activation of $\mathrm{Na}+-\mathrm{K}+-2 \mathrm{Cl}$ - cotransporter facilitates 
332 glioma migration. Mol Cancer. 2014. 13: 31.

333 25. Ma H, Li T, Tao Z, Hai L, Tong L, Yi L, Abeysekera IR, Liu P, Xie Y, Li J, Yuan F, Zhang

334 C, Yang Y, Ming H, Yu S, Yang X. NKCC1 promotes EMT-like process in GBM via RhoA and

335 Rac1 signaling pathways. J Cell Physiol, 2019; 234: 1630-42.

336 26. Haas BR, Cuddapah VA, Watkins S, Rohn KJ, Dy TE, Sontheimer H. With-No-Lysine

337 Kinase 3 (WNK3) stimulates glioma invasion by regulating cell volume. Am J Physiol Cell

338 Physiol, 2011; 301: C1150-60.Figure Legend

339

340

341

342

343

344

345

346

347

348

349

350

351

352

353

354

355

356

357

358

359

360

361

362

363

364

365

366

367

368

369

370

Figure 1: Impact of NKCC1 expression on overall survival in Mesenchymal GBM patients in TCGA cohort.

Figure 2: Correlations and validations of EMT markers and NKCC1 in GBM. The Pearson Correlation between NKCC1 and EMT markers, including (A) CDH2, (B) VIM, (C) Zeb1 and (D) CTNNB1 in the TCGA GBM dataset.

Figure 3: Enrichment plots from Gene Set Enrichment Analysis (GSEA). GSEA results showing that (A) (B) gliomas, MAPK signaling pathways, TGF-beta signaling and epithelial mesenchymal transitions are differentially enriched in NKCC1-related GBM.

Figure 4: NKCC1 expression associated with the histopathological grade in human glioma tissues. Images of immunohistochemical staining of NKCC1 in human glioma tissues. (A) Grade I (B) Grade II (C) Grade III (D) Grade IV

Figure 5: NKCC1 promoted the ability of U251 and U87 cells to migrate and invade. (A-D) Transwell assay showing migration in the U87 cell line (E-H) Transwell assay showing invasion in the U87 cell line (I-L) Transwell assay showing migration in the U251 cell line (M-P) Transwell assay showing invasion in the U87 cell line (* indicates $\mathrm{p}<0.05$ )

Figure 6: Silencing of NKCC1 in U87 and U251 as detected by western blotting. $\beta$-actin was used as a positive control. Vim, Zeb1, CDH2, CTNNB1, NKCC1, MMP2 and MMP9 were detected by western blotting after knockdown of NKCC1. (A-B) Representative immunoblots for the expression of Vim, Zeb1, CDH2, CTNNB1, NKCC1, MMP2 and MMP9 in U87 and U251 cells $(\mathrm{C}-\mathrm{H})$ Summary data of immunoblotting expression of each protein was first normalized by $\beta$-actin and relative expression levels in U87 and U251 cells (* indicates $p<0.05$ )

Figure 7: Silencing of NKCC1 in U87 and U251 as detected by western blotting. $\beta$-actin was used as a positive control. CTNNB1(Catenin beta-1) was detected by western blotting after knockdown of NKCC1. (A) Representative immunoblots for the expression of CTNNB1 in U87 and U251 cells (B) Summary data of immunoblotting expression 
371 of CTNNB1 first normalized by $\beta$-actin and the relative expression level in U87 and U251 cells $372 \quad(*$ indicates $\mathrm{p}<0.05)$

373

374 
Figure 1

survival

Impact of NKCC1 expression on overall survival in Mesenchymal GBM patients in TCGA cohort

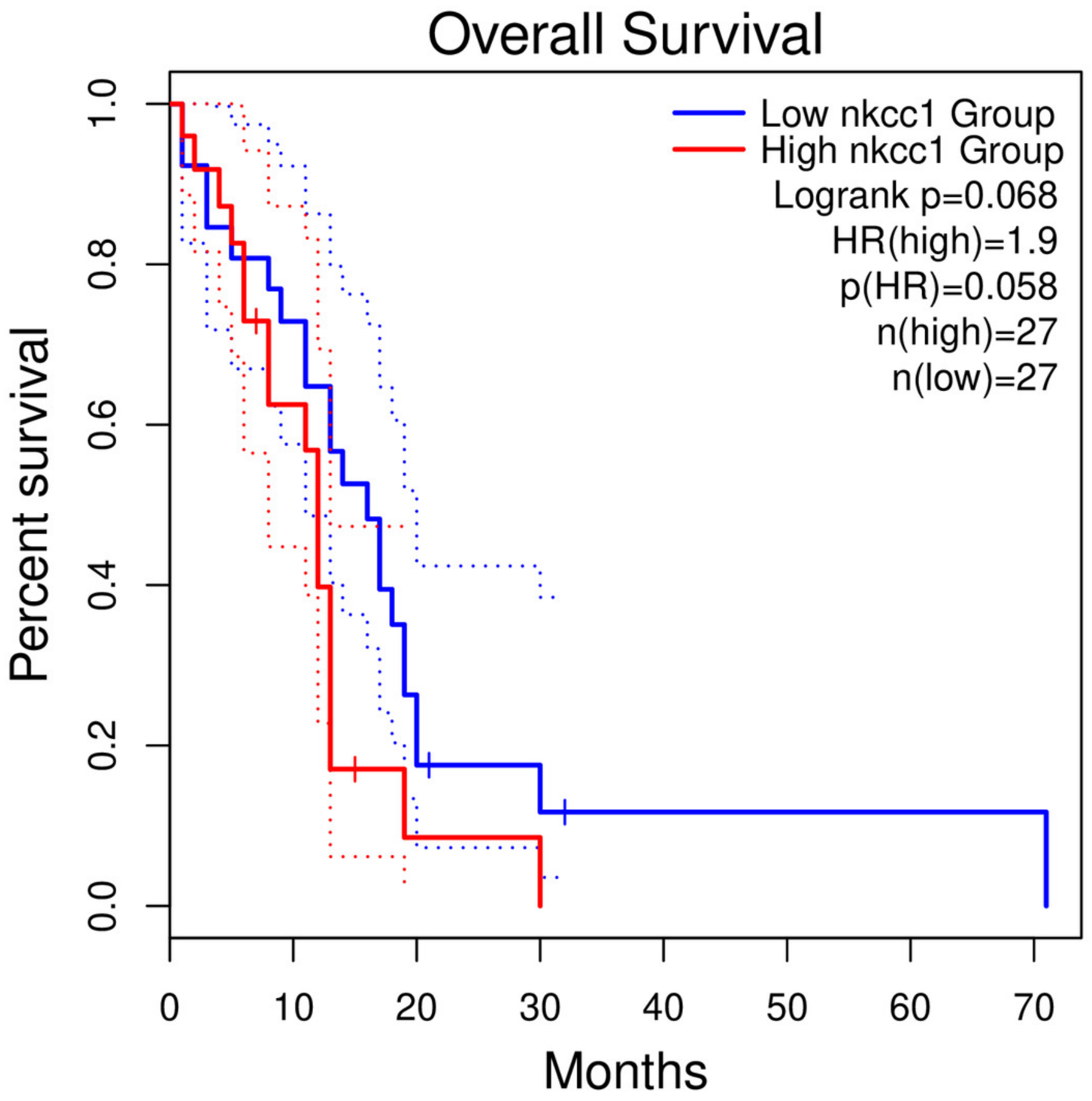


Figure 2

\section{EMT markers}

Correlations and validations of EMT markers with NKCC1 in GBM. The Pearson correlations between NKCC1 and the EMT markers, including (A) CDH2, (B) VIM, (C)Zeb1 and (D)CTNNB1 in the TCGA GBM dataset.
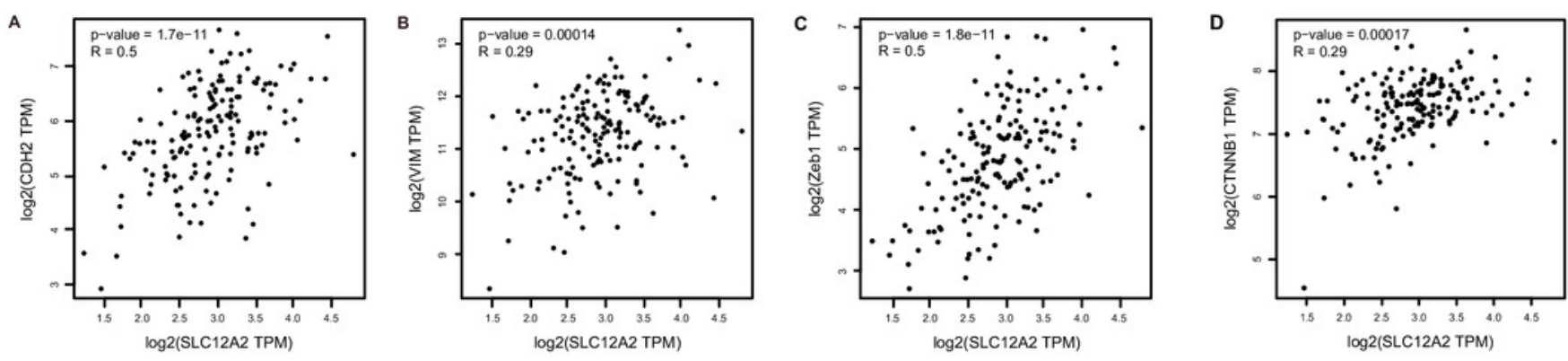
Figure 3

\section{GSEA}

Enrichment plots from gene set enrichment analysis (GSEA). GSEA results showing (A) (B) glioma, MAPK signaling pathway, TGF-beta signaling, Epithelial Mesenchymal Transition are differentially enriched in NKCC1-related GBM

A

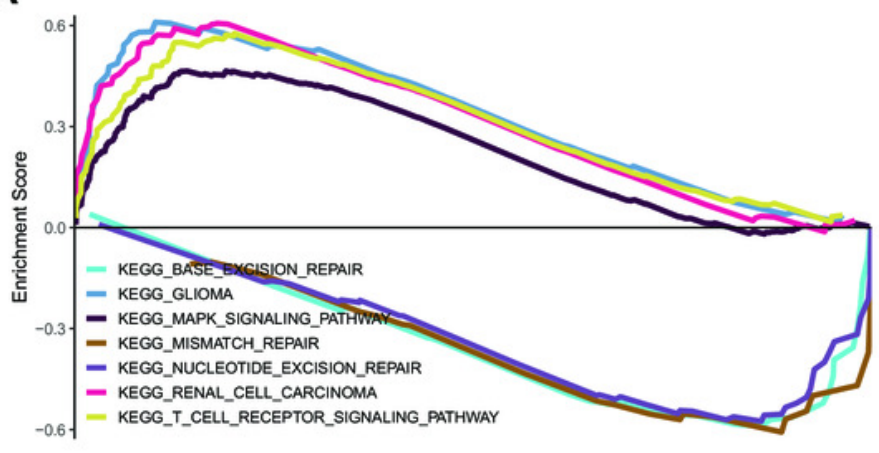

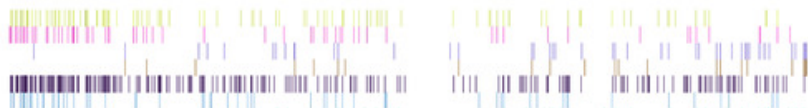

high expression<----------->low expression
B
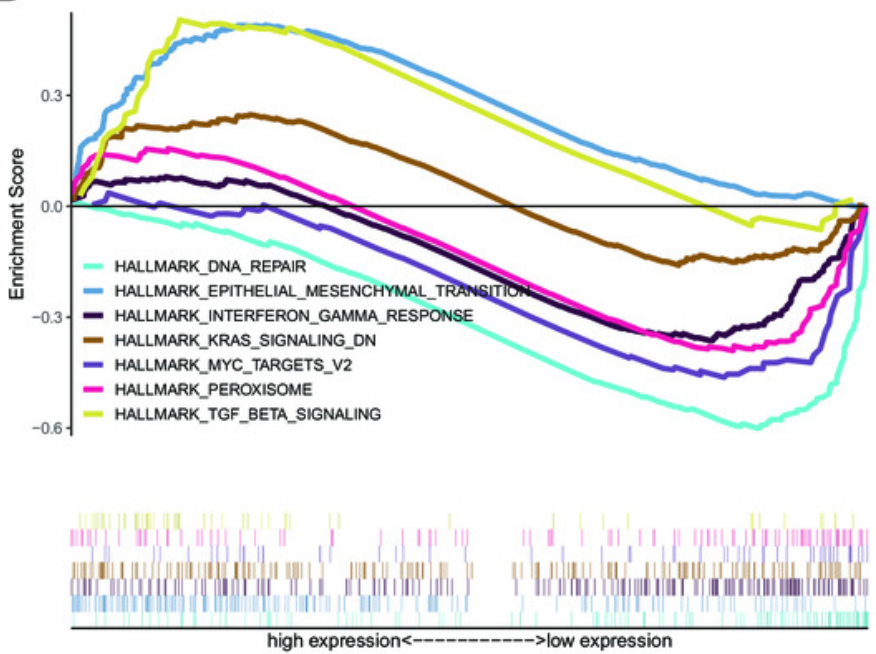


\section{Figure 4}

$\mathrm{IHC}$

NKCC1 expression was associated with the histopathological grade in human glioma tissues.

The images of immunohistochemical staining of NKCC1 in human different grade glioma tissues. (A) is the picture of Grade I glioma tissue. (B) is Grade II. (C) is Grade III. (D) is Grade

IV.

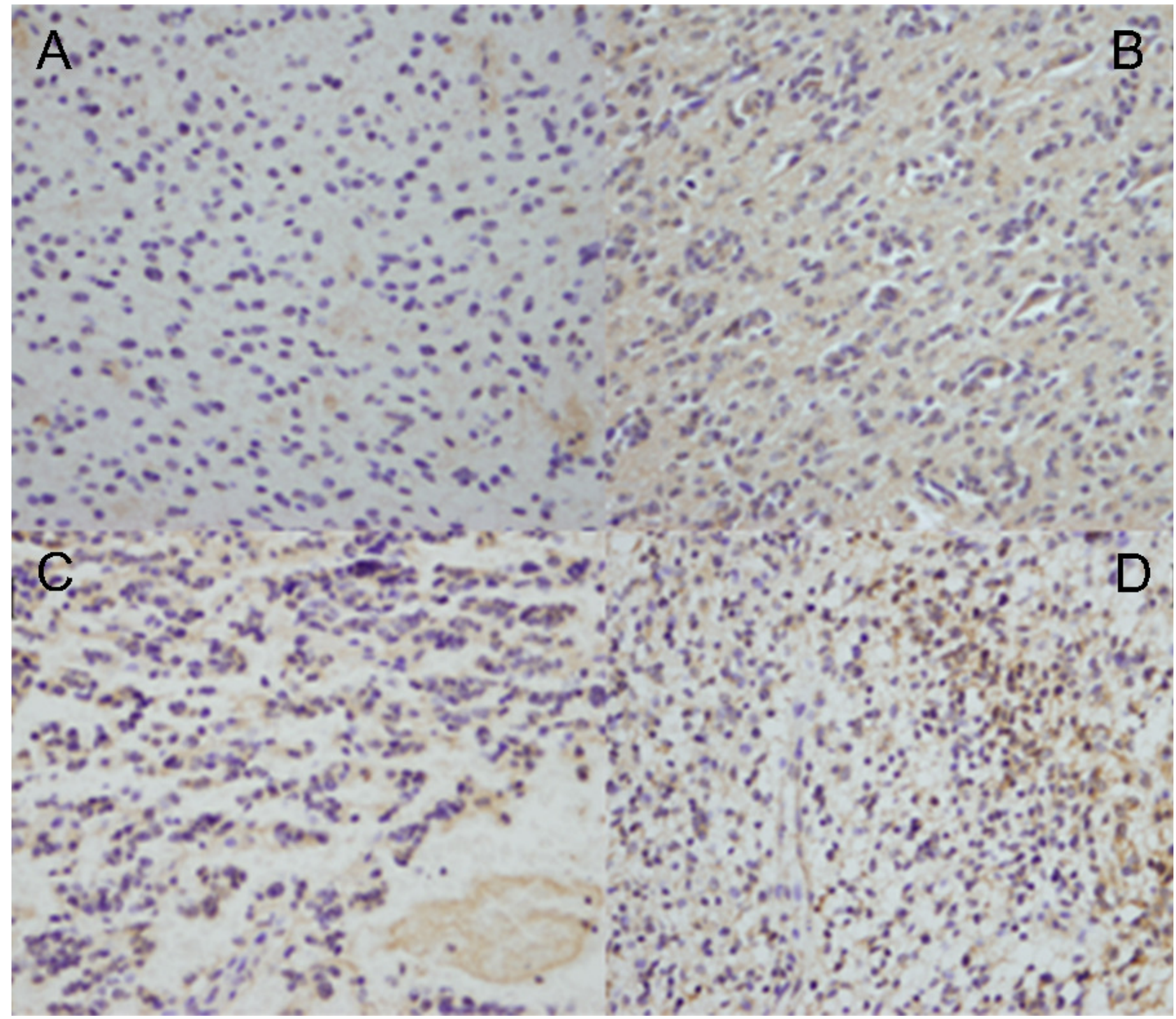




\section{Figure 5}

\section{Transwell}

NKCC1 promoted the ability of U251 and U87 cells to migrate and invade. (A-D) Transwell assay showing migration in U87 cell line. (E-H) Transwell assay showing invasion ability in U87 cell line. (I-L) Transwell assay showing migration ability in U251 cell line. (M-P) Transwell assay showing invasion ability in U87 cell line. * indicates $\mathrm{P}<0.05$. 

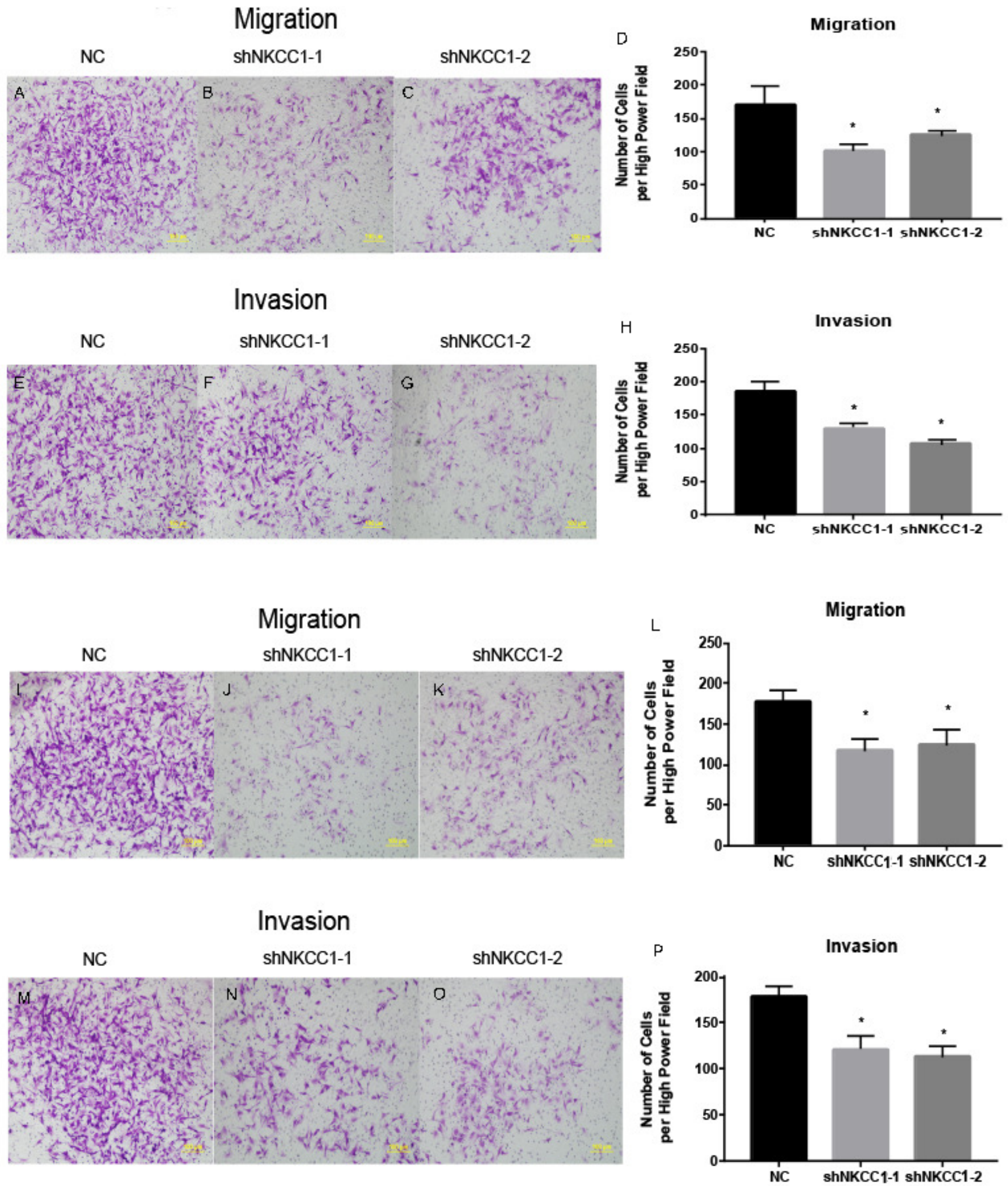


\section{Figure 6}

Western

Silencing of NKCC1 in U87 and U251 as detected by western blotting. $\beta$-actin was used as a positive control. Vim, Zeb1, CDH2, CTNNB1, NKCC1, MMP2 and MMP9 were detected by western blotting after knockdown of NKCC1. (A-B) Representative immunoblots for expression of Vim, Zeb1, CDH2, CTNNB1, NKCC1, MMP2 and MMP9 in U87 and U251 cell. (CH) Summary data of immunoblotting expression of each protein was first normalized by $\beta$ actin and relative expression level in U87 and U251 cell. * indicates $\mathrm{P}<0.05$. 
A
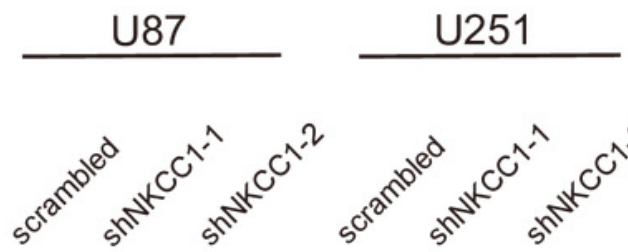

Vim

Zeb1

$\mathrm{CDH} 2$

$\beta$-actin
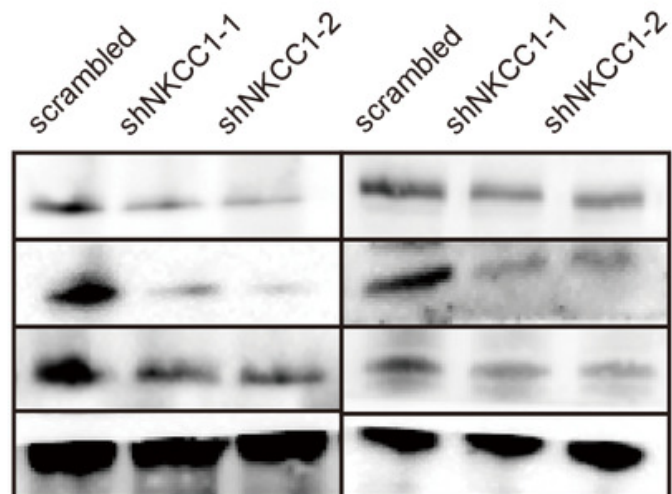

B

U87

NKCC1

MMP2

MMP9

$\beta$-actin
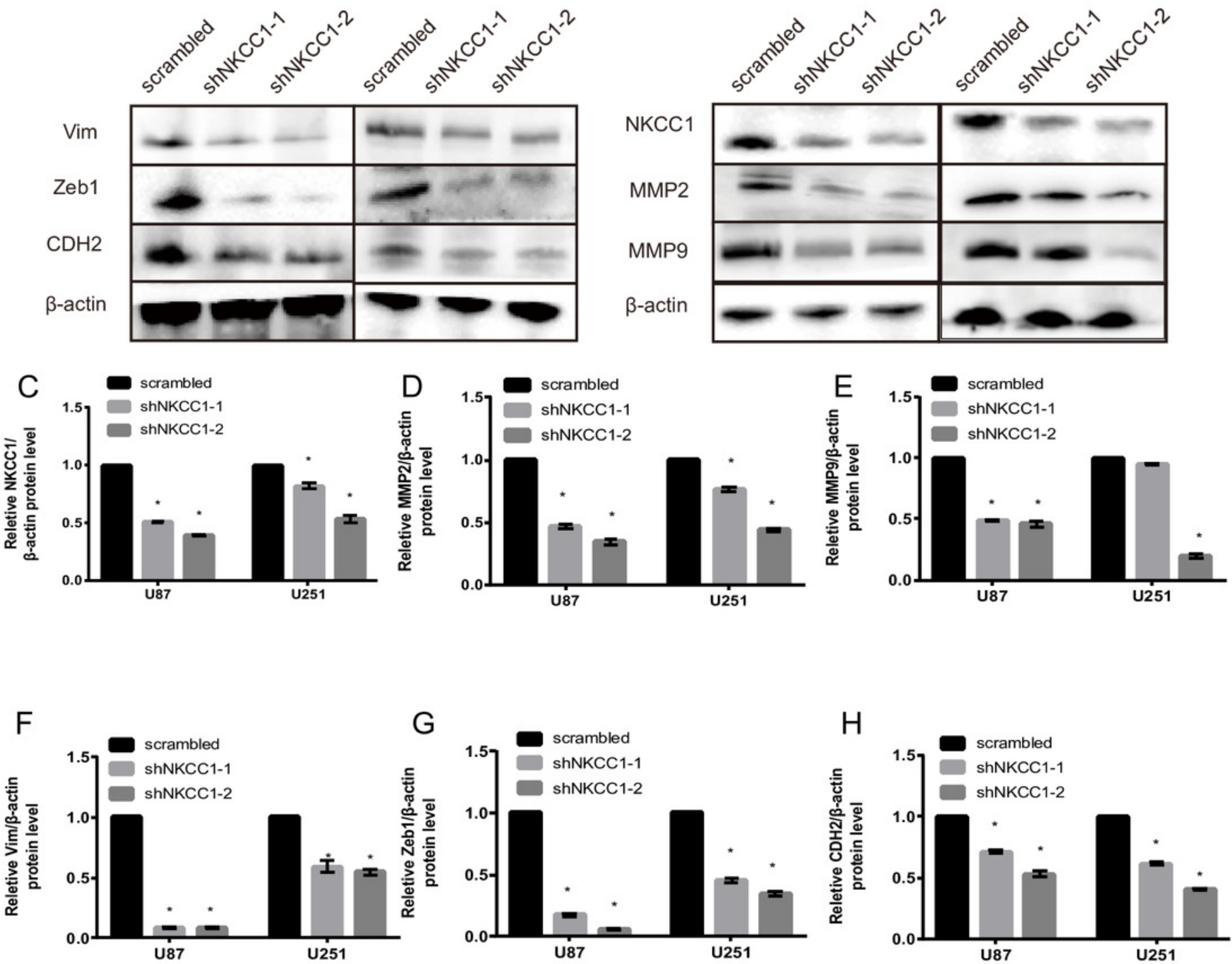


\section{Figure 7}

Western

Silencing of NKCC1 in U87 and U251 as detected by western blotting. $\beta$-actin was used as a positive control. CTNNB1(Catenin beta-1) was detected by western blotting after knockdown of NKCC1. (A) Representative immunoblots for expression of CTNNB1 in U87 and U251 cell. (B) Summary data of immunoblotting expression of CTNNB1 was first normalized by $\beta$-actin and relative expression level in U87 and U251 cell. * indicates $\mathrm{P}<0.05$. 
A

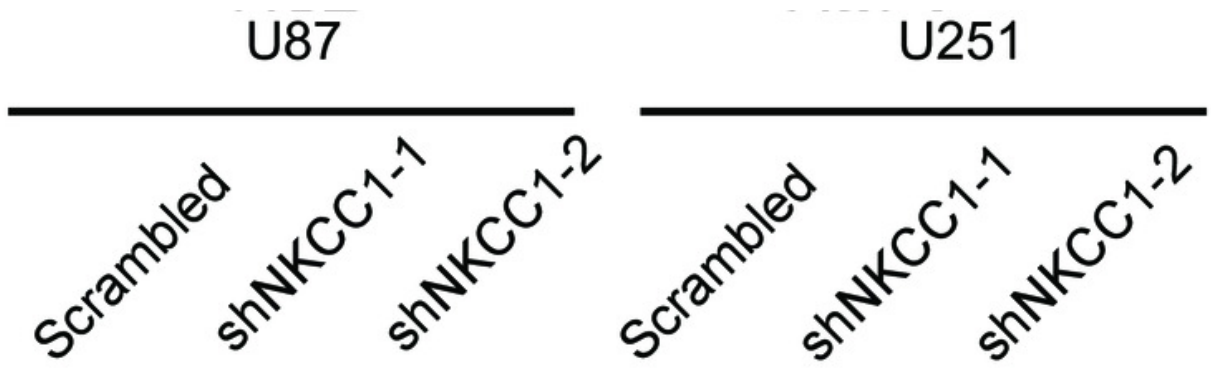

CTNNB1

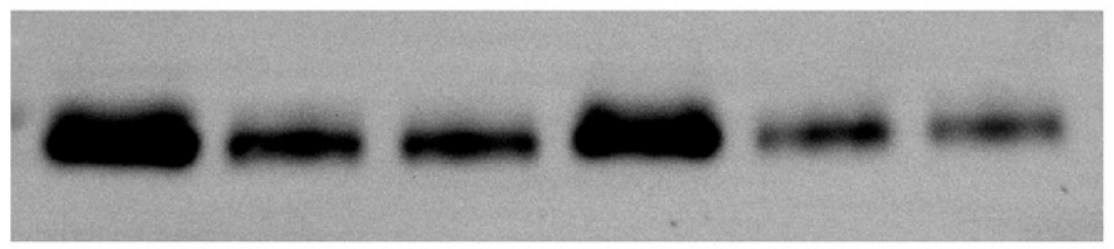

$\beta$-actin

B

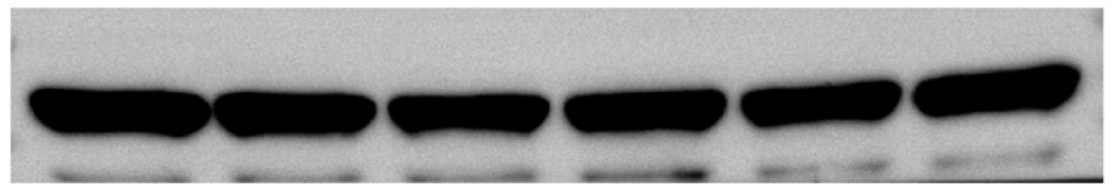

scrambled

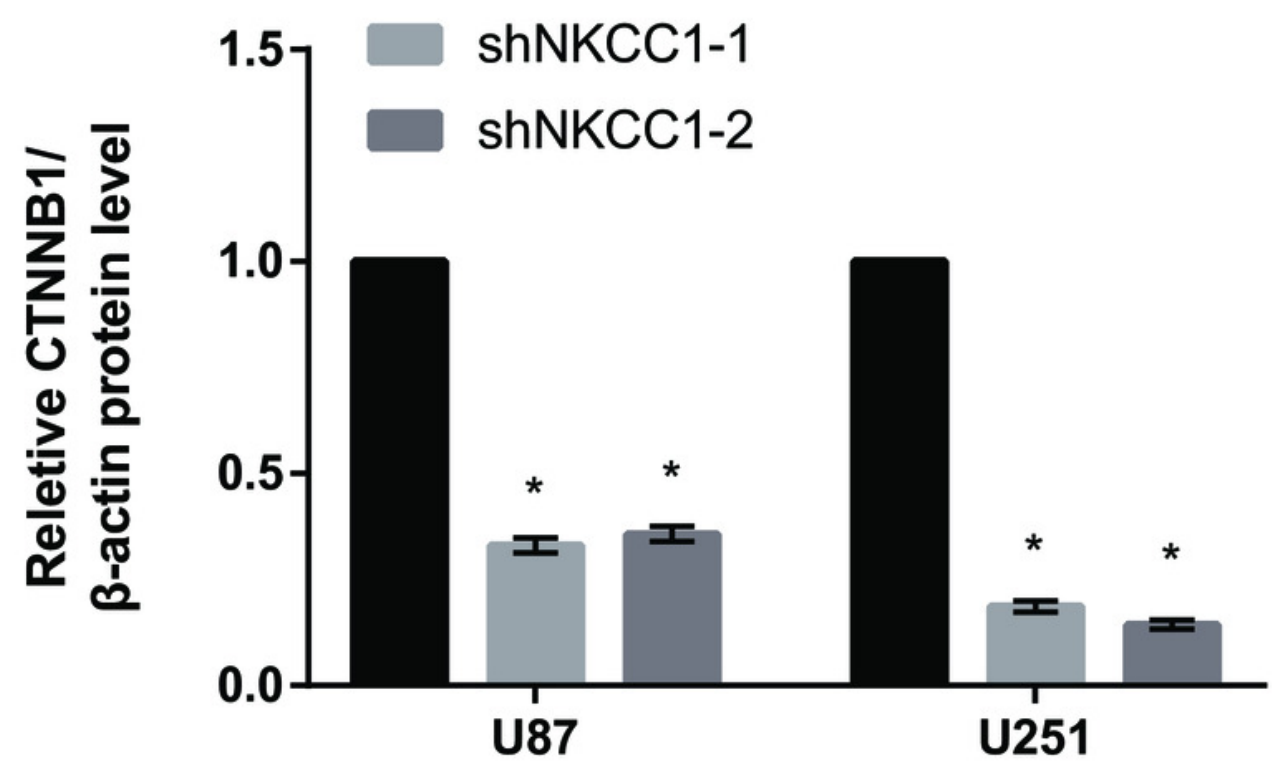


Table $\mathbf{1}$ (on next page)

$\mathrm{IHC}$

Clinicopathological characteristics of glioma patients based on NKCC1 expression status 
Clinicopathological characteristics of glioma patients based on NKCC1 expression status

\begin{tabular}{|c|c|c|c|c|c|c|}
\hline & \multirow{2}{*}{ Characteristic } & \multicolumn{4}{|c|}{ IHC intensity scoring } & \multirow{2}{*}{$p$ value } \\
\hline & & 0 & +1 & +2 & +3 & \\
\hline \multirow{2}{*}{ Sex } & Male & 4 & 8 & 5 & 10 & \multirow{2}{*}{0.277} \\
\hline & Female & 3 & 7 & 3 & 21 & \\
\hline \multirow{2}{*}{ Age } & $<50$ & 5 & 6 & 6 & 14 & \multirow{2}{*}{0.445} \\
\hline & $\geq 50$ & 2 & 3 & 7 & 17 & \\
\hline \multirow{4}{*}{ Grade } & I & 1 & 3 & 0 & 1 & \multirow{4}{*}{0.001} \\
\hline & II & 4 & 9 & 3 & 3 & \\
\hline & III & 2 & 1 & 4 & 9 & \\
\hline & IV & 0 & 2 & 1 & 18 & \\
\hline \multirow{3}{*}{ Location } & Left cerebral hemisphere & 1 & 8 & 4 & 15 & \multirow{3}{*}{0.62} \\
\hline & Right cerebral hemisphere & 6 & 7 & 4 & 15 & \\
\hline & Cerebellum & 0 & 0 & 0 & 1 & \\
\hline
\end{tabular}

